\title{
Identidad profesional docente en aspirantes a profesorado de enseñanza secundaria
}

\author{
${ }^{1}$ Alfonso Pontes, ${ }^{2}$ Leopoldo Ariza y ${ }^{3}$ Rosario Del Rey \\ ${ }^{1}$ Máster FPES de Tecnología, IDEP, Universidad de Córdoba \\ ${ }^{2}$ Departamento de Educación Artística y Corporal, Universidad de Córdoba \\ ${ }^{3}$ Departamento de Psicología Evolutiva y de la Educación, Universidad de Sevilla \\ (Recibido 22 de Junio 2010; Aceptado 23 de Septiembre 2010)
}

RESUMEN: Dentro de la línea de investigación sobre el pensamiento de los docentes, en este trabajo se muestran los resultados de un estudio destinado a explorar las ideas y motivaciones de los futuros profesores de educación secundaria sobre la profesión docente y la formación pedagógica necesaria para ser profesor en esta etapa educativa. Mediante el Cuestionario sobre interés por la docencia y la formación inicial (Pontes, Ariza y Sánchez, 2010) se han explorado las opiniones de 353 alumnos y alumnas del curso de formación inicial para la docencia, que constituyen aproximadamente la cuarta parte de los estudiantes de la Universidad de Córdoba en los dos pasados cursos, es decir, los últimos que han cursado el CAP y los primeros que han realizado el Máster de Profesorado de Educación Secundaria. Entre los resultados se ha encontrado que los motivos de interés profesional por la docencia obedecen a diferentes causas y que están relacionados con distintas concepciones previas sobre la profesión y la formación docente. Los resultados obtenidos contribuyen al avance de la investigación sobre el desarrollo inicial de la identidad profesional docente entre los profesores y profesoras en formación y ofrecen claves para la mejora del proceso de formación inicial del profesorado de secundaria.

\section{Teaching professional identity of candidates for secondary school teachers}

ABSTRACT: Within the line of investigation over teacher's thinking, we show with this paper the results obtained from a research which aim was to explore the ideas and motivation of future secondary school teachers towards the teacher profession and the pedagogic training needed for being teacher in this educational stage. Through the Questionnaire about interest to teaching and initial training (Pontes, Ariza y Sánchez, 2010) we have explored the opinions of 353 students of the course of initial training for teachers, comprising a quarter of the students of the past two courses of the University of Cordoba and being the last students of the CAP and the first of the Master degree. Among the results we have found that professional interests towards teaching obey to different reasons and that these reasons are related to diverse prior conceptions about the teacher profession and its training. The results obtained contribute to the advance of the investigation about initial development of the teaching professional identity of training teachers and provide with some keys for the improvement of the initial training process of the secondary school teachers.

Palabras clave: Formación inicial, motivos por la docencia, concepciones previas 
Key words: Initial training, motivation towards training, prior conceptions.

\section{INTRODUCCIÓN}

Los futuros profesores y profesoras de enseñanza secundaria poseen actitudes, motivaciones e ideas previas sobre la docencia y sobre la formación inicial (Campanario, 1998) que influyen en la formación de la identidad profesional docente (Bolívar, 2006; Marcelo, 2009) y que deben tenerse en cuenta en los procesos de formación del profesorado, para mejorar el diseño instruccional y las actividades de enseñanza-aprendizaje a realizar en tales procesos (Day, 2005; González Gallego, 2010). Por ello, es conveniente aprovechar el momento actual de adaptación del modelo de formación inicial docente al Espacio Europeo de Educación Superior, que ha supuesto la sustitución del antiguo curso del Certificado de Aptitud Pedagógica (CAP) por el nuevo Máster de Profesorado de Enseñanza Secundaria (MPES), para profundizar en la línea de investigación sobre el pensamiento inicial de los futuros profesores, que incluye desde el estudio de las actitudes, expectativas e intereses hasta las concepciones previas o teorías implícitas sobre un amplio número de temas relacionados con el ejercicio de la profesión docente (Prosser, Martin, Trigwell, Ramsden y Lueckenhausen, 2005; Hernández y Maquilón, 2010; Porlán, Martín, Rivero, Harres, Azcárate y Pizzato, 2010).

En estudios previos sobre el pensamiento inicial docente, se ha observado que existe un amplio espectro de motivaciones que se conjugan, según los distintos momentos formativos y tipo de participantes, incentivando en distinta medida el interés por la docencia de las muestras analizadas (Córdoba, Ortega y Pontes, 2006). El interés profesional por la docencia es un tema de estudio relevante en relación con el papel que desempeñan las motivaciones en el proceso de formación y en el desarrollo de la identidad profesional docente (Esteve, 1997), ya que se han observado motivaciones de tipo altruista y humanista, ligadas al papel positivo que desempeña la educación en la sociedad, que pueden considerarse como el inicio de una vocación docente o motivación intrínseca (Varela y Ortega, 1984), que coexisten con otros de naturaleza más pragmática hacia el ejercicio de la profesión docente, donde priman los estereotipos acerca de los beneficios materiales de la profesión como son el salario, las vacaciones, etc. (Pontes y Serrano, 2008; Sánchez Lissen, 2009).

Por otra parte, la motivación por la profesión docente entre los aspirantes a profesores de enseñanza secundaria es un tema que está muy relacionado con el interés por adquirir una formación inicial adecuada para el ejercicio de esta compleja profesión (Pérez, Gilar y González, 2007). Con relación al tema de la formación inicial docente se han publicado números trabajos de carácter teórico y crítico, centrados sobre todo en formular propuestas metodológicas y curriculares para la mejora del proceso de formación (Darling-Hammond y Bransford, 2005; Korthagen, Loughrany Russell, 2006) y también se han realizado estudios empíricos orientados a recoger las opiniones de los futuros profesores de secundaria sobre diversos aspectos del proceso de formación inicial (García y Martínez, 
2001; Fuentes, García y Martínez, 2009) o las necesidades formativas de los profesores noveles o principiantes (Pro, Valcárcel y Sánchez, 2005; Marcelo, 2009).

En tales estudios se ha observado que los futuros profesores y profesoras de secundaria presentan diferentes formas de entender y valorar la formación inicial, aunque la mayoría coincide en que la docencia es una profesión compleja y que se necesita una buena formación inicial para su ejercicio. También se ha visto que muchos sujetos valoran más la formación sobre didáctica específica y las prácticas docentes que la formación en aspectos psicopedagógicos de carácter general, o que los sujetos con mayor interés profesional por la docencia son los que presentan mayores demandas de formación, tanto en los temas a tratar como en la extensión del periodo de formación.

Con objeto de profundizar en esta línea de trabajo se ha llevado a cabo un estudio sobre las motivaciones hacia la docencia y las opiniones sobre la formación docente, entre futuros profesores de enseñanza secundaria al comenzar el proceso de formación inicial, en poblaciones de sujetos que participan en diferentes procesos de formación (CAP y MPES). Tal estudio se ha realizado en varias fases, utilizando diferentes instrumentos, que han permitido recoger datos sobre el pensamiento inicial en el alumnado del curso del CAP y hacer una primera valoración de la utilidad de tales instrumentos (Pontes et al., 2010). En la última fase, cuyos resultados se muestran en este trabajo, se ha encuestado también a un conjunto amplio de alumnos y alumnas del nuevo Máster de Formación del Profesorado de Educación Secundaria Obligatoria y Bachillerato, Formación Profesional y Enseñanza de Idiomas (MPES).

El objetivo principal de este nuevo estudio es conocer el grado de interés por la profesión docente y el tipo de motivos que caracterizan ese acercamiento a la docencia, el momento en que surgen tales intereses, los factores internos o externos que han influido en esa motivación y las ideas previas sobre la formación inicial necesaria para el ejercicio de la docencia en la educación secundaria, ya que entendemos que todos estos aspectos están relacionados con el desarrollo inicial de la identidad profesional docente, que es un tema de gran interés para la investigación educativa y la formación inicial del profesorado (Day, 2005; Marcelo, 2009). Como objetivo complementario de este estudio se ha tratado de valorar si existen diferencias significativas entre las motivaciones, actitudes e ideas previas del alumnado del curso del CAP y del MPES en torno a los aspectos abordados en el cuestionario, ya que el CAP era un curso masificado y poco profesionalizador, al que accedía una población muy amplia, mientras que al nuevo máster acede un conjunto algo más restringido de sujetos a los que se les puede suponer una mayor motivación por la formación y la profesión docente (Vilches y Gil, 2010).

\section{MÉTODO}

\section{Participantes}

En este estudio se han recogido datos de dos conjuntos de participantes inte- 
grados por un total de 353 estudiantes del curso de formación inicial de profesorado de enseñanza secundaria de la Universidad de Córdoba, correspondientes a dos modelos o procesos de formación diferentes. El primer grupo estaba integrado por un total de 218 sujetos, 80 varones y 138 mujeres, alumnos del CAP durante el curso 2008-2009, con una edad media de 26.4 años (DT=3,48) y el segundo por 145 sujetos, 54 alumnos y 81 alumnas de la primera edición del Máster de Profesorado de Enseñanza Secundaria (MPES) durante el curso 20092010, con una edad media de 27.3 años (DT $=3,46$ ).

\section{Instrumento}

Partiendo de los resultados de un estudio exploratorio anterior, donde se usaron cuestiones abiertas para conocer las ideas previas de un grupo de 67 alumnos del CAP de la Universidad de Córdoba (Pontes y Serrano, 2008), se diseñó el Cuestionario sobre interés por la docencia y la formación inicial-CIDFI- (Pontes et al., 2010) que se incluye en el Anexo I de este trabajo. El CIDFI está compuesto por 19 ítems valorados en un escala Likert de 4 grados de acuerdo y distribuidos en dos apartados: Interés por la docencia (12 ítems) e Ideas previas sobre la formación docente ( 7 ítems) más datos generales como la edad, el género y la especialidad del curso de formación inicial docente.

\section{Procedimiento}

El primer día de clase presencial se entregaba a todo el alumnado el CIDFI que fue contestado de manera voluntaria y anónima. Tras la recogida y tabulación de datos, se procedió a la recodificación y análisis de los mismos usando el paquete estadístico SPSS (Versión 15.0) y realizando análisis de medias, frecuencias y porcentajes.

\section{RESULTADOS}

\section{Interés por la docencia}

El alumnado encuestado afirma tener interés profesional por ejercer la docencia ( $M=3.07$; DT: 0.757$)$, aunque el origen de ese interés puede responder a motivaciones de diferente tipo: ausencia de otras salidas profesionales $(\mathrm{M}=1.92$; DT: 0.938), interés por las condiciones materiales del trabajo docente $(\mathrm{M}=2.28$; DT: 0.900$)$, atracción por la educación y por trabajar con jóvenes $(\mathrm{M}=3.22$; DT: 0.714). Sin embargo, dicho interés se produce en diferentes momentos de la vida: previo a los estudios universitarios $(\mathrm{M}=2.29$; DT: 1.038$)$, al finalizar los estudios ( $\mathrm{M}=2.46$; DT: 1.039) y se ve influido por el recuerdo positivo de antiguos profesores $(M=2.98$; DT: 0.985), los antecedentes familiares $(M=2.25$; DT: 1.285$)$ o una percepción positiva de la profesión docente en su círculo social $(\mathrm{M}=1.71$; DT: 0.837). 
En cuanto a las posibles diferencias y similitudes entre las poblaciones de CAP y MPES, aparece un notable paralelismo en los resultados de ambas poblaciones ya que existe bastante similitud entre los valores medios de cada ítem a excepción de la creencia sobre que en la actualidad los docentes tienen mayores problemas (ver tabla 1).

\begin{tabular}{|c|c|c|c|c|c|c|}
\hline ITEMS & $\begin{array}{l}\text { MODELO } \\
\text { FIP }\end{array}$ & $\mathrm{N}$ & Media & $\begin{array}{l}\text { Desviación } \\
\text { típica }\end{array}$ & $\begin{array}{c}\mathrm{T} \text { de } \\
\text { Student }\end{array}$ & $\underset{\text { (signif) }}{\mathrm{p}}$ \\
\hline \multirow[t]{2}{*}{ A1 } & CAP & 218 & 3,04 &, 730 & & \\
\hline & MPES & 135 & 3,11 & 798 &,- 879 & 089 \\
\hline \multirow[t]{2}{*}{$\mathrm{A} 2$} & CAP & 218 & 1.89 & 944 & & \\
\hline & MPES & 135 & 1,96 & 929 &,- 641 & 136 \\
\hline \multirow{2}{*}{$\mathrm{A} 3$} & CAP & 218 & 2,30 & 1,016 & & \\
\hline & MPES & 135 & 2,27 & 1,075 & 249 & 256 \\
\hline \multirow[t]{2}{*}{$\mathrm{A} 4$} & CAP & 218 & 2.36 & 984 & & \\
\hline & MPES & 135 & 2,64 & 1,104 & $-2,407$ &,- 051 \\
\hline \multirow[t]{2}{*}{ A5 } & CAP & 218 & 2,18 & 860 & & \\
\hline & MPES & 135 & 2,44 & 943 & $-2,593$ &,- 061 \\
\hline \multirow[t]{2}{*}{ A6 } & CAP & 218 & 3,21 & .726 & & \\
\hline & MPES & 135 & 3,24 & .696 &,- 431 & 119 \\
\hline \multirow[t]{2}{*}{ A7 } & CAP & 218 & 2,86 &, 952 & & \\
\hline & MPES & 135 & 2,81 & 918 & ,494 & 251 \\
\hline \multirow[t]{2}{*}{$\mathrm{A} 8$} & CAP & 218 & 3,21 & .724 & & \\
\hline & MPES & 135 & 3,27 & .747 &,- 836 & 092 \\
\hline \multirow[t]{2}{*}{ A9 } & CAP & 218 & 2,88 & 1,009 & & \\
\hline & MPES & 135 & 3,11 & .944 & $-2,170$ &,$- 021 *$ \\
\hline \multirow[t]{2}{*}{ A10 } & CAP & 218 & 3,01 & 965 & & \\
\hline & MPES & 135 & 2,93 & 1,019 & ,761 & 299 \\
\hline \multirow[t]{2}{*}{ A11 } & CAP & 218 & 1,72 &, 836 & & \\
\hline & MPES & 135 & 1,69 & .842 &, 390 & 217 \\
\hline \multirow[t]{2}{*}{$\mathrm{A} 12$} & CAP & 218 & 2,22 & 1,292 & & \\
\hline & MPES & 135 & 2,30 & 1,277 & $\begin{array}{l}-, 562 \\
\end{array}$ & 198 \\
\hline
\end{tabular}

Tabla 1: Comparación de valores medios sobre Interés por la docencia

En relación a la percepción sobre la profesión docente, los sujetos encuestados parecen ser conscientes de los problemas actuales de la enseñanza secundaria $(\mathrm{M}=2.84$; DT: 0.938$)$ y de la complejidad de la profesión docente actual $(\mathrm{M}=3.23$; DT: 0.733$)$ así como de que ellos serán profesores con más problemas que aquellos que los educaron a ellos $(\mathrm{M}=2.97$; DT: 0.990). Con el objetivo de profundizar en el análisis de las motivaciones hacia la docencia se establece una dimensión Interés por la docencia, integrada por cinco nuevas variables, definidas a partir de las anteriores: a) Actitud vocacional, gran interés por la docencia y compromiso para superar los problemas; b) Actitud pragmática, señalan la necesidad de trabajo o énfasis en las condiciones materiales; c) Origen temprano del interés por la docencia; d) Imagen positiva de la docencia; e) Influencia externa, relevancia del influjo familiar o de antiguos profesores. Los resultados generales respecto a la valoración de la profesión docente, teniendo en cuenta los valores bastante y mucho, muestran que el $46.8 \%$ tiene una imagen positiva de la docencia, el $41.3 \%$ señala influencias externas, el $40.5 \%$ muestra un interés 
pragmático, el 30.6\% manifiesta un interés temprano por la docencia, y el 27.7\% apunta hacia un interés eminentemente vocacional. En la tabla 2 se detallan los resultados descriptivos globales, diferenciados según el modelo de formación inicial.

\begin{tabular}{|c|c|c|c|c|c|c|}
\hline Motivos y Modelo d & ormación & $\begin{array}{l}\text { Nada } \\
\% \text { (f) }\end{array}$ & $\begin{array}{l}\text { Poco } \\
\% \text { (f) }\end{array}$ & $\begin{array}{c}\text { Bastante } \\
\% \text { (f) }\end{array}$ & $\begin{array}{l}\text { Mucho } \\
\%(\mathrm{f})\end{array}$ & Total \\
\hline \multirow[t]{3}{*}{ Interés vocacional } & CAP & $50,5(110)$ & $24,3(53)$ & $15,1(33)$ & $9,9(22)$ & 218 \\
\hline & MPES & $43,0(58)$ & $25,2(34)$ & $22,2(30)$ & $10,1(13)$ & 135 \\
\hline & Total & $47,7(168)$ & $24,5(87)$ & $17,8(63)$ & $9,9(35)$ & 353 \\
\hline \multirow[t]{3}{*}{ Interés pragmático } & CAP & $40,4(88)$ & $22,9(50)$ & $18,3(40)$ & $18,3(40)$ & 218 \\
\hline & MPES & $34,1(46)$ & $19,3(26)$ & $25,2(34)$ & $21,5(29)$ & 135 \\
\hline & Total & $40(134)$ & $21,5(76)$ & $21(74)$ & $19,5(69)$ & 353 \\
\hline \multirow[t]{3}{*}{ Origen temprano } & CAP & $44,0(96)$ & $25,2(55)$ & $12,4(27)$ & $18,3(40)$ & 218 \\
\hline & MPES & $56,8(75)$ & $12,1(16)$ & $15,2(20)$ & $15,9(21)$ & 132 \\
\hline & Total & $48,5(171)$ & $21(71)$ & $13,3(47)$ & $17,3(61)$ & 350 \\
\hline \multirow[t]{3}{*}{ Imagen positiva } & CAP & $29,4(63)$ & $20,1(43)$ & $36,9(79)$ & $13,6(29)$ & 214 \\
\hline & MPES & $32,1(42)$ & $24,4(32)$ & $32,1(42)$ & $11,5(15)$ & 131 \\
\hline & Total & $29,7(105)$ & $21,2(75)$ & $34,3(121)$ & $12,5(44)$ & 345 \\
\hline \multirow[t]{3}{*}{ Influencia externa } & CAP & $34,4(75)$ & $26,6(58)$ & $13,8(30)$ & $25,2(55)$ & 218 \\
\hline & MPES & $31,9(43)$ & $23,0(31)$ & $21,5(29)$ & $23,7(32)$ & 135 \\
\hline & Total & $33,4(118)$ & $25 ’ 2(89)$ & $16,7(59)$ & $24,6(87)$ & 353 \\
\hline
\end{tabular}

Tabla 2. Motivos para la docencia según modalidad de formación

\section{Ideas previas sobre la formación docente inicial}

Entre las ideas previas de los estudiantes de formación inicial para la profesión docente, las más apoyadas son aquellas relacionadas con formación psicoeducativa, ya que según la población encuestada para ser profesor de secundaria es necesario adquirir importantes conocimientos de carácter psicopedagógico y didáctico $(\mathrm{M}=3.19 ; \mathrm{DT}=0.915)$ y sobre programación docente $(\mathrm{M}=3.52 ; \mathrm{DT}=0.670)$ y conceden gran importancia a la necesidad de adquirir destrezas docentes que permitan motivar a los alumnos por el aprendizaje $(\mathrm{M}=3.25$; DT=0.713). En menor medida, pero también con valores considerables, señalan ideas relacionadas con la necesidad de adquirir un conocimiento amplio de los contenidos y de saber transmitirlos con claridad $(\mathrm{M}=2.40 ; \mathrm{DT}=0.958)$, la importancia de la experiencia en la formación docente $(\mathrm{M}=2.49 ; \mathrm{DT}=0.829)$, o de las cualidades innatas para la comunicación y la docencia $(=2.47 ; \mathrm{DT}=0.965)$ y la minusvaloración de la formación psicoeducativa $(\mathrm{M}=2.40 ; \mathrm{DT}=0.851)$. Con relación a las posibles diferencias entre los grupos de formación inicial (CAP o MPES) las tendencias son similares en las respuestas emitidas por ambos grupos, sin que existan diferencias significativas entre ellos, a excepción de la misnuvaloración de la formación 
psicoeducativa (ver tabla 3).

\begin{tabular}{llccccc}
\hline ITEMS & MODELO FIPS & $\mathrm{N}$ & Media & $\begin{array}{c}\text { Desviación } \\
\text { Típica }\end{array}$ & $\begin{array}{c}\mathrm{Tde} \\
\text { Student }\end{array}$ & $\begin{array}{c}\mathrm{p} \\
\text { (signif) }\end{array}$ \\
\hline B1 & CAP & 218 & 2,05 &, 763 & & \\
\hline & MPES & 135 & 2,97 &, 969 & $-9,907$ &,- 737 \\
\hline B2 & CAP & 218 & 3,36 &, 725 & & \\
\hline & MPES & 135 & 2,93 & 1,111 & 4,420 &, 624 \\
\hline B3 & CAP & 218 & 2,32 &, 807 & & \\
\hline & MPES & 135 & 2,54 &, 904 & $-2,423$ &,$- 042^{*}$ \\
\hline B4 & CAP & 217 & 3,34 &, 625 & & \\
\hline B5 & MPES & 135 & 3,12 &, 820 & 2,815 &, 370 \\
\hline & CAP & 218 & 2,53 &, 901 & & \\
\hline B6 & MPES & 135 & 2,37 & 1,056 & 1,477 &, 377 \\
\hline & CAP & 218 & 3,56 &, 636 & & \\
\hline B7 & MPES & 135 & 3,47 &, 721 & 1,231 &, 242 \\
\hline & CAP & 218 & 2,39 &, 848 & & \\
\hline & MPES & 135 & 2,64 &, 779 & $-2,748$ &,- 069 \\
\hline
\end{tabular}

Tabla 3: Comparación de valores medios en ideas previas de la formación docente.

Para el análisis global de las ideas sobre la formación docente inicial se establece una dimensión integrada por cuatro nuevas variables: a) Modelo transmisivo, cuando se da prioridad del conocimiento de los contenidos a impartir y la capacidad para transmitirlos; b) Formación integral, relevancia del dominio psicoeducativo; c) Formación empírica, la práctica como epicentro del proceso de formación, infravalorando la formación psicoeducativa; d) Cualidades innatas, cuando asumen la existencia de una capacidad individual previa para la comunicación y la docencia. Los resultados descriptivos muestran que el alumnado encuestado no se caracteriza por situarse en una única forma de definir las claves de la formación inicial. Así, el 44.2\% apoyaría bastante o mucho la formación integral, el $40.2 \%$ el modelo transmisivo, el $39.6 \%$ las cualidades innatas y el $33,7 \%$ la formación empírica (ver tabla 4).

\begin{tabular}{llccccc}
\hline Claves y Modelo de Formación & $\begin{array}{c}\text { Nada } \\
\%(\mathrm{f})\end{array}$ & $\begin{array}{c}\text { Poco } \\
\%(\mathrm{f})\end{array}$ & $\begin{array}{c}\text { Bastante } \\
\%(\mathrm{f})\end{array}$ & $\begin{array}{c}\text { Mucho } \\
\%(\mathrm{f})\end{array}$ & Total \\
\hline Modelo trasmisivo & CAP & $23,4(51)$ & $41,7(91)$ & $22,0(48)$ & $13,4(28)$ & 218 \\
& MPES & $17,0(23)$ & $34,7(46)$ & $31,6(43)$ & $17,0(23)$ & 135 \\
& Total & $20,9(74)$ & $38,8(137)$ & $25,8(91)$ & $14,4(51)$ & 353 \\
\hline Formación integral & CAP & $30,0(65)$ & $23,5(51)$ & $20,7(45)$ & $25,8(56)$ & 217 \\
& MPES & $43,0(58)$ & $14,1(19)$ & $20,7(28)$ & $22,2(30)$ & 135 \\
& Total & $34,8(123)$ & $19,8(70)$ & $20,1(73)$ & $24,4(86)$ & 353 \\
\hline Formación empírica & CAP & $50,9(111)$ & $19,7(43)$ & $19,3(42)$ & $10,1(22)$ & 218 \\
& MPES & $31,9(43)$ & $27,4(37)$ & $25,2(34)$ & $15,6(21)$ & 135 \\
& Total & $43,6(154)$ & $22,7(80)$ & $21,5(76)$ & $12,2(43)$ & 353 \\
\hline
\end{tabular}




\begin{tabular}{llccccc}
\hline Cualidades innatas & CAP & $9,6(21)$ & $43,1(94)$ & $29,8(65)$ & $17,0(37)$ & 218 \\
& MPES & $14,1(19)$ & $37,8(51)$ & $27,4(37)$ & $16,3(22)$ & 135 \\
& Total & $11,3(40)$ & $41,1(145)$ & $22,9(102)$ & $16,7(59)$ & 353 \\
\hline
\end{tabular}

Tabla 4. Claves para la formación docente según modalidad de formación

\section{DISCUSIÓN Y CONCLUSIONES}

El estudio realizado apoya algunas tendencias y características del pensamiento inicial -sobre la profesión docente y las motivaciones hacia la docenciaobservadas en estudios anteriores realizados con otras técnicas de exploración (Córdoba, et al., 2006; Pérez et al., 2007; Pontes y Serrano, 2008).

Los resultados del estudio señalan que las motivaciones hacia la docencia y concepciones sobre la formación inicial son bastante similares entre el alumnado del CAP y del MPES, lo cual ya se puso de manifiesto en otro estudio sobre el pensamiento inicial docente (Fernández, Elortegui y Medina, 2002) de alumnos del CAP y del Curso de Cualificación Pedagógica (CCP), que es un curso similar en extensión y contenidos al nuevo Máster de formación de profesorado. Este resultado permite pensar que los alumnos del CAP y del MPES encuestados en este estudio representan muestras diferentes de una misma población, ya que poseen unos estudios universitarios similares y acceden al curso de formación inicial docente con actitudes e ideas previas bastante parecidas.

El interés por la docencia de este colectivo puede ser de tipo vocacional o de carácter pragmático y su origen se sitúa en distintos momentos del itinerario formativo: al comenzar los estudios universitarios o más comúnmente al finalizar los estudios o al comenzar a buscar trabajo. Estas motivaciones y tendencias ya han sido señaladas en estudios anteriores, pero los datos cuantitativos reflejan diferencias respecto a estudios sobre las motivaciones por la docencia entre estudiantes de magisterio (Sánchez Lissen, 2009) o entre estudiantes universitarios de carreras de letras (Córdoba et al., 2006) y de ciencias (Córdoba, Ortega y Pontes, 2009).

Cerca de la mitad de los estudiantes muestran una imagen positiva de la docencia en la educación secundaria, pese a ser conscientes de los problemas históricos y emergentes de la profesión, lo cual contrasta con estudios que reflejan una visión algo más pesimista (García y Martínez, 2001; Pontes y Serrano, 2008) y queda en evidencia el rol influyente de las experiencias personales y familiares para desarrollar el interés por esta profesión, hecho también previamente descrito (Campanario, 1998).

Sorprendentemente, la idea de que es necesario adquirir una formación inicial docente integrada por conocimientos psicopedagógicos, didácticos y prácticos es asumida solo por casi la mitad de los sujetos y, tal y como se ha descrito (Martínez Losada, García y Mondelo, 1993; Pérez et al., 2007), se infravalora la formación psicopedagógica inicial considerando importante la transmisión de conocimientos disciplinares, las cualidades innatas para la docencia o la expe- 
riencia como verdadera formación docente. Estos datos nos inducen a considerar que hay un colectivo de estudiantes opuestos a la necesidad de adquirir una sólida formación inicial docente, lo que hace más difícil aún si cabe esta formación tan importante para la calidad de la educación secundaria obligatoria. Como se ha observado en otros trabajos previos (Campanario, 1998; Sánchez Lissen, 2009), algunas de las actitudes o concepciones previas sobre la profesión y la formación docente muestran relación respecto al género, la edad y la titulación de los sujetos encuestados, pero este tema se abordará en un estudio posterior.

Entre las conclusiones e implicaciones derivadas de este estudio debemos destacar la necesidad de tener en cuenta los resultados obtenidos en este tipo de investigaciones a la hora de diseñar el proceso de formación inicial de los futuros profesores de secundaria, tanto por parte de los responsables académicos como de los profesores que imparten docencia en el nuevo máster de profesorado de secundaria (Vílchez y Gil, 2010). Sobre todo, sería necesario incluir en el diseño curricular del máster un tratamiento específico del tema de la identidad profesional docente, ligado al conocimiento didáctico del contenido y al desarrollo del currículum educativo de cada especialidad (Beijard, Meijer y Verloop, 2004; Bolivar, 2006).

Por otra parte, desde el punto de vista metodológico, los profesores de las diferentes materias del máster deberían diseñar y desarrollar actividades que permitan explicitar las ideas previas y motivaciones de los futuros docentes, en torno a cualquier aspecto relacionado con el ejercicio de la actividad docente (Hernández y Maquilón, 2010). Al mismo tiempo sería conveniente seguir profundizando en la investigación sobre el pensamiento inicial docente de los profesores en formación, tratando de analizar la relación entre las actitudes hacia la docencia y las concepciones sobre los procesos educativos, para ayudar a desarrollar competencias docentes ligadas a la progresión de las ideas y motivaciones previas (Darling-Hammond y Bransford, 2005; Porlán et al., 2010).

Para finalizar, señalar que los resultados del estudio deben ser tomados con cautela debido a sus limitaciones relativas especialmente al tamaño de la muestra y la naturaleza del instrumento de recogida. Sin embargo, a pesar de ellas consideramos que la información aportada puede ser una línea base desde la que desplegar análisis más complejos y procesos de investigación más minuciosos.

\section{Agradecimientos}

Los autores agradecen al Instituto de Estudios de Postgrado (IDEP) de la Universidad de Córdoba por favorecer el acceso a los estudiantes encuestados

\section{REFERENCIAS BIBLIOGRÁFICAS}

Beijaard, D., Meijer, P. C., y Verloop, N. (2004). Reconsidering research on teachers professional identity. Teaching and Teacher Education, 20(2), 107-128. 
Bolivar, A. (2006). La identidad profesional del profesorado de de secundaria: Crisis y reconstrucción. Archidona: Aljibe.

Campanario, J.M. (1998). ¿Quiénes son, qué piensan y qué saben los futuros maestros y profesores de ciencias?: una revisión de estudios recientes. Revista Interuniversitaria de Formación del Profesorado, 33, 121-140.

Córdoba, F., Ortega, R., y Pontes, A. (2006). La docencia en educación secundaria como salida profesional para el alumnado de Humanidades y Ciencias Sociales: un estudio sobre intereses y motivos. Revista de Investigación Educativa, 24(2), 519-540.

Córdoba, F., Ortega, R., y Pontes, A. (2009). Universitarios de Ciencias ante la docencia en Educación Secundaria como expectativa profesional. Revista de Educación, 348, 401-421.

Darling-Hammond, L., y Bransford, J. (Eds.) (2005). Preparing teachers for a changing world. San Francisco: Jossey Bass.

Day, C. (2005). Pasión por enseñar. La identidad personal y profesional del docente y sus valores. Madrid: Narcea.

Esteve, J.M. (1997). La formación inicial de los profesores de secundaria. Una reflexión sobre el curso de cualificación pedagógica. Barcelona: Ariel Educación.

Fernández, J., Elortegui, N., y Medina, M. (2002). Formación de profesorado de ciencias de la naturaleza, de educación secundaria, a partir de sus ideas previas. Investigación en la Escuela, 47, 65-74.

Fuentes, M.J.; García, S., y Martínez, C. (2009). ¿En qué medida cambian las ideas de los futuros docentes de Secundaria sobre qué y cómo enseñar, después de un proceso de formación? Revista de Educación, 349, 269-294.

García Barros, S., y Martínez Losada, C. (2001). Las ideas de los alumnos del CAP, punto de referencia para reflexionar sobre formación docente. Revista Interuniversitaria de Formación del Profesorado, 40, 97-110.

González Gallego, I. (Coord.) (2010). El nuevo profesor de secundaria: La formación inicial docente en el marco del espacio europeo de educación superior. Barcelona: Graó.

Hernández, F., y Maquilón, J. (2010). Las concepciones de la enseñanza. Aportaciones para la formación del profesorado. Revista Electrónica Interuniversitaria de Formación del Profesorado, 13 (3), 17-25.

Korthagen, F., Loughran, J., y Russell, T. (2006). Developing fundamental principles for teacher education programs and practices. Teaching and Teacher Education, 22(8), 1.020-1.041.

Marcelo, C. (2009). Formalidad e Informalidad en el oficio de aprender a enseñar. Revista de Educación. 350, 31-55.

Martínez Losada, C., García, S., y Mondelo, M. (1993). Las ideas de los profesores de ciencias sobre la formación docente. Enseñanza de las Ciencias, 11 (1), 26-32.

Pérez, A.M., Gilar, R. y González, C. (2007). Pensamiento y formación del profesorado de educación secundaria. Revista Electrónica de Investigación Psi- 
coeducativa, las Ciencias, 5(2), 307-324.

Pontes, A., Ariza, L., y Sánchez, F.J. (en prensa). Ideas previas sobre la profesión docente y la formación inicial entre futuros profesores de enseñanza secundaria. ED.UCO: Revista de investigación educativa, 6.

Pontes, A., y Serrano, R. (2008). Actitudes e ideas previas sobre la docencia y la formación docente en los aspirantes a profesores de ciencias experimentales. En R. Jiménez Liso (Eds.), Ciencias para el mundo contemporáneo y formación del profesorado en didáctica de las ciencias experimentales (pp. 458-467). Almería: Editorial Universidad de Almería.

Porlán, R., Martín, R., Rivero, A., Harres, J., Azcárate, P., y Pizzato, M. (2010). El cambio del profesorado de ciencias I: marco teórico y formativo. Enseñanza de las Ciencias, 28 (1), 31-46.

Pro, A., Valcárcel, M.V., y Sánchez, G. (2005). Viabilidad de las propuestas didácticas planteadas en la formación inicial: opiniones, dificultades y necesidades de profesores principiantes. Enseñanza de las Ciencias, 23 (3), 357378.

Prosser, M., Martin, E., Trigwell, K. Ramsden, P., y Lueckenhausen, G. (2005). Academics Experiences of Understanding of their Subject Matter and the Relationship to their Experiences of teaching and learning. Instructional Science, 33, 137-157.

Sánchez Lissen, E (2009). Mitos y realidades en la carrera docente. Revista de Educación, 348, 465-488.

Varela, J., y Ortega, F. (1984). El aprendiz de maestro. Madrid: MEC.

Vilches, A., y Gil, D. (2010). Máster de Formación Inicial del Profesorado de Enseñanza Secundaria. Algunos análisis y propuestas. Revista Eureka sobre enseñanza y divulgación de las ciencias, 7(3), 661-666. 


\section{ANEXO I: CUESTIONARIO SOBRE INTERÉS POR LA DOCENCIA Y LA FORMACIÓN INICIAL}

Datos Iniciales: a) Género: ; b) Edad: c) Especialidad:

Indicar (entre 1 y 4 ) el grado de acuerdo con las siguientes proposiciones

\begin{tabular}{|c|c|c|c|c|}
\hline A) INTERÉS POR LA DOCENCIA & \begin{tabular}{|c|}
$(1)$ \\
Nada
\end{tabular} & $\begin{array}{c}\text { (2) } \\
\text { Poco }\end{array}$ & $\begin{array}{c}\text { (3) } \\
\text { Bastante }\end{array}$ & $\begin{array}{c}\text { (4) } \\
\text { Mucho }\end{array}$ \\
\hline \multicolumn{5}{|l|}{$\begin{array}{l}\text { A1. Tengo verdadero interés profesional por ejercer la docencia en Educación } \\
\text { Secundaria }\end{array}$} \\
\hline \multicolumn{5}{|l|}{$\begin{array}{l}\text { A2. Solo me interesa la docencia porque no tengo otras salidas profesionales } \\
\text { o es difícil acceder a ellas. }\end{array}$} \\
\hline \multicolumn{5}{|l|}{$\begin{array}{l}\text { A3. Mí interés por la docencia proviene desde que comencé los estudios } \\
\text { universitarios. }\end{array}$} \\
\hline \multicolumn{5}{|l|}{$\begin{array}{l}\text { A4. Durante los estudios universitarios aspiraba a otra profesión pero ahora } \\
\text { la docencia me parece interesante }\end{array}$} \\
\hline \multicolumn{5}{|l|}{$\begin{array}{l}\text { A5. La docencia en secundaria sólo interesa por sus condiciones materiales } \\
\text { de trabajo: estabilidad, vacaciones,... }\end{array}$} \\
\hline \multicolumn{5}{|l|}{$\begin{array}{l}\text { A6. La profesión docente es agradable y motivadora por el hecho de trabajar } \\
\text { educando a personas jóvenes }\end{array}$} \\
\hline \multicolumn{5}{|l|}{$\begin{array}{l}\text { A7. Los tiempos actuales no ofrecen una imagen muy buena de la profesión } \\
\text { docente en secundaria. }\end{array}$} \\
\hline \multicolumn{5}{|l|}{$\begin{array}{l}\text { A8. La profesión docente es problemática pero se pueden superar las dificultades } \\
\text { y aprender a ser buen profesor/a. }\end{array}$} \\
\hline \multicolumn{5}{|l|}{$\begin{array}{l}\text { A9. Cuando yo era estudiante de secundaria los profesores tenían menos } \\
\text { problemas para enseñar que ahora. }\end{array}$} \\
\hline \multicolumn{5}{|l|}{$\begin{array}{l}\text { A10. Algunos de mis profesores/as han ejercido una influencia positiva para } \\
\text { interesarme por la docencia }\end{array}$} \\
\hline \multicolumn{5}{|l|}{$\begin{array}{l}\text { A11. En mi círculo social no existe una buena opinión sobre la profesión } \\
\text { docente en secundaria. }\end{array}$} \\
\hline \multicolumn{5}{|l|}{$\begin{array}{l}\text { A12. Mi interés por la docencia tiene antecedentes familiares y personas } \\
\text { conocidas que están contentas con su profesión }\end{array}$} \\
\hline \multicolumn{5}{|l|}{ B) FORMACIÓN DOCENTE } \\
\hline \multicolumn{5}{|l|}{$\begin{array}{l}\text { B1. Para ser profesor/a solo importa tener un amplio conocimiento de los } \\
\text { contenidos y enseñarlos con claridad }\end{array}$} \\
\hline \multicolumn{5}{|l|}{$\begin{array}{l}\text { B2. Antes de ser profesor/a es necesario adquirir conocimientos profundos de } \\
\text { psicología, pedagogía y didáctica específica de la propia materia que se ha de } \\
\text { enseñar }\end{array}$} \\
\hline \multicolumn{5}{|l|}{$\begin{array}{l}\text { B3. La formación docente del profesor no se adquiere mediante cursos de } \\
\text { pedagogía o de didáctica }\end{array}$} \\
\hline \multicolumn{5}{|l|}{$\begin{array}{l}\text { B4. Para motivar a los alumnos por el aprendizaje es necesario adquirir ciertas } \\
\text { destrezas propias de la profesión docente }\end{array}$} \\
\hline \multicolumn{5}{|l|}{$\begin{array}{l}\text { B5. Hay personas con cualidades innatas para la comunicación y la docencia, } \\
\text { pero tales destrezas no se adquieren en cursos de formación didáctica }\end{array}$} \\
\hline \multicolumn{5}{|l|}{$\begin{array}{l}\text { B6. El profesorado de secundaria, además de conocer bien su materia, debe } \\
\text { aprender a programar su actividad docente y adquirir destrezas adecuadas para } \\
\text { dominar las técnicas e instrumentos de comunicación efectiva en el aula }\end{array}$} \\
\hline B7. Sólo se aprende a ser buen profesor a través de la experiencia & & & & \\
\hline
\end{tabular}

